\title{
NOTE ON NAMES, ORTHOGRAPHY AND PRONUNCIATION
}

Africans who live in Lesotho generally refer to themselves as Basotho. The singular is Mosotho. The language, culture and way of life are referred to as Sesotho. In the colonial period, the usages Basuto or Basutos were common, but are used here only in direct quotation.

The Sesotho language has two orthographies, one used in Lesotho, the other in South Africa. We use the Lesotho orthography in this book. It has several peculiarities. (1) A double consonant, as in ho lla (to cry), is invariably a prolongation of the single consonant. The double $\mathrm{Mm}$ in the prefix of a woman's name is written 'Ma-, as in 'Mantšebo. (2) When placed before another vowel $e$ is often pronounced as $y$. (3) An $l$ before an $i$ or a $u$ is pronounced as $d$. So liretlo is pronounced diretlo and Lerotholi is pronounced Lerothodi. (4) When placed before another vowel $o$ is often pronounced as $w$. So Moshoeshoe is pronounced Moshweshwe. (5) ph represents an aspirated $p$; th an aspirated $t$; and š an aspirated $s$. 